\title{
On Welding Residual Stresses near Fatigue Crack Tips
} \author{
Jonas Hensel ${ }^{1, a^{*}}$, Thomas Nitschke-Pagel ${ }^{1, b}$ and Klaus Dilger ${ }^{1, c}$ \\ ${ }^{1}$ Institute of Joining and Welding, TU Braunschweig, Langer Kamp 8, 38106 Braunschweig, \\ Germany \\ aj.hensel@tu-braunschweig.de, bt.pagel@tu-braunschweig.de, 'k.dilger@tu-braunschweig.de \\ * corresponding author
}

Keywords: Residual stresses, fatigue, crack, neutron diffraction, longitudinal welded gusset.

\begin{abstract}
Residual stresses in steel plates with longitudinal fillet welded gussets have been experimentally determined by means of neutron diffraction. The redistribution of the residual stresses due to fatigue crack growth has been studied. The residual stress fields determined have been related to experimental fatigue strength data in terms of constant amplitude fatigue testing data (S-N data). Further, the fatigue crack region has been characterized by means of metallographic investigations such as micrographs, Vickers hardness measurements and computerized tomography.

The presented work is on welded specimens, made of unalloyed fine grained carbon steel, with different fatigue crack lengths. The results of the diffraction measurement show that the initial tensile residual stresses near the weld toe are, caused by phase transformation at low temperatures, below the base material's yield strength. Tensile residual stresses in the order of the yield strength can be found below the material's surface. These areas of high residual stresses move with the propagating crack tip through the specimen during fatigue loading.
\end{abstract}

\section{Introduction}

Welding is commonly used for joining of cyclically loaded steel or aluminum structures. These structures must be investigated regarding their fatigue strength. The fatigue strength of welded structures is affected by welding residual stresses, influencing the crack propagation rate [1]. The crack propagation rate is a measure of fracture mechanics and often investigated in small scale $\mathrm{C}(\mathrm{T})$ (compact tension) samples. This sample type is fairly small and thus macro residual stresses are rather low [2]. Samples with higher residual stresses, for instance welded steel plates, allow studying residual stress effects on crack propagation in larger scale. Such investigations on welded plates have been published by different authors, examples of which are given in [1-4].

For instance, the residual stress effect on crack propagation was observed by [1] for linear deposit welds. These welds were made of unalloyed mild steels and the residual stresses were determined with a sectioning method using strain gauges. The determined residual stresses were simplified and used for crack propagation calculations without taking the redistribution into account.

[2] have carried out investigations on butt welded plates of aluminum alloy (2219-T851). For this alloy, [2] showed that tensile residual stresses, acting in the same direction as load stresses, increase the crack propagation significantly. Similar results from investigations on longitudinal deposit welds, made of an aluminum alloy (2024-T351), have been published by [3]. [3] also pointed out that the elastic redistribution of the initial residual stress field has significant effect on the crack propagation rate [4]. Residual stress effects in such welded plates were found to be comparable to mean stresses in C(T) tests. Further it was mentioned that observed compressive residual stresses in the wake of the crack, however, are resulting from a measurement error. As a reason for this, it was mentioned that the compressive residual stresses are not affected by applied loads.

The linear weld shape is the common characteristic of the aforementioned fundamental investigations on residual stress effects on crack propagation. Linear welds with high residual stresses in longitudinal weld direction are well suited for this kind of fundamental investigations. In 
real welded structures this weld detail is not as critical as, for instance, welds transverse to the loading direction.

The weld geometry presented in this work is also associated with high tensile residual stresses and it can rather be found in real welded components. It is a fatigue-critical weld detail with potential to dominate the fatigue resistance design of an entire component. For this specimen type investigations on the residual stress redistribution due to fatigue crack growth have not been published yet and will be presented in this work.

\section{Experimental setup}

Welded constructions under fatigue load are often designed based on a simple nominal stress approach. The experimental basis for this design approach is commonly established based on small scale specimen tests. The specimen type used in this investigation is a steel plate with a non-load carrying longitudinal fillet weld, see Fig. 1. That fillet weld leads to change in stiffness and causes under mechanical loading a stress concentration. The highest stress concentration can be expected at the weld toe. This is where a fatigue crack starts under cyclic loads.

One characteristic of this small scale specimen type is the shrinkage constraint for the cooling weld in longitudinal sample direction. This shrinkage constraint is the requirement for high tensile residual stresses. Although high tensile residual stresses can be expected below the weld, it has been shown that residual stresses at the weld toe are reduced by the austenite phase transformation [5]. The effect of the reduction of tensile residual stresses by austenite phase transformation is associated with the cooling conditions [6] and can be observed here in the heat affected zone (HAZ). This zone is approximately $2 \mathrm{~mm}$ wide and deep starting from the weld toe. However, the high tensile residual stresses below the weld allow studying the behavior of the residual stress field with a growing fatigue crack.

Specimen preparation. The material used for the specimens was an unalloyed fine-grained mild construction steel (steel grade 1.0546) with a yield strength $\left(\mathrm{R}_{\mathrm{e}}\right)$ of $360 \mathrm{MPa}$. This material is widely used in many engineering disciplines for welded constructions.

The specimens were manufactured with a robot-mounted metal active gas (MAG) welding torch. The filler metal used for specimen preparation was EN ISO 14341-A-G4Si1. The dimensions of the base plate were $440 \times 80 \mathrm{~mm}^{2}$ with a thickness of $12 \mathrm{~mm}$, compare Fig. 1 (a). The one-sided longitudinal attachment had dimensions of $150 \times 30 \mathrm{~mm}^{2}$. The start-stop of the weld seam was located at the long side of the attachment.

Fatigue cracks were generated at the weld toe of the fillet weld (Fig. 1 (b)) under alternating tension-compression loads with constant amplitudes $(\mathrm{R}=-1)$. The chosen load level $\Delta \sigma=120 \mathrm{MPa}$ corresponds to a fatigue life $\mathrm{N}$ in the finite life region $\left(\mathrm{N}<2 \times 10^{6}\right)$. In particular, the samples were loaded to a pre-defined number of load cycles and stopped resulting in different fatigue crack lengths at the weld toe. The chosen load cycles were $\mathrm{N}=3 \mathrm{E} 5$ respectively $\mathrm{N}=4.5 \mathrm{E} 5$.

(a) Specimen geometry

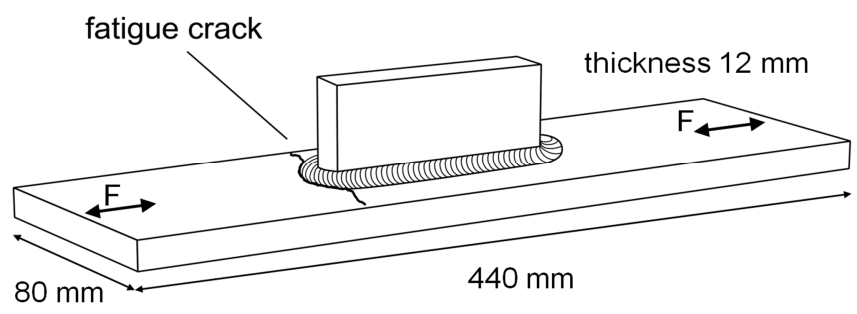

(b) Close up of the fatigue crack

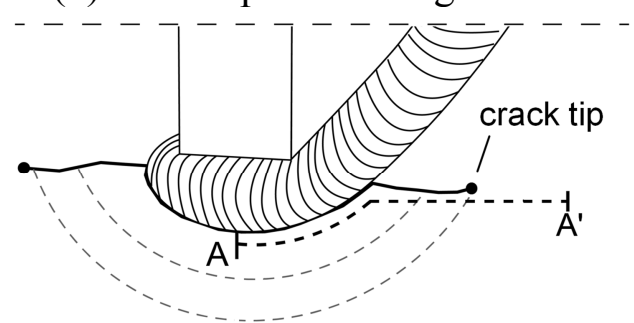

Fig 1 (a) Specimen geometry and (b) location of fatigue crack initiation; (b) shows the path A-A' used for Neutron diffraction measurement

The neutron diffraction measurement was concentrated on the cross section containing the fatigue crack. The crack path followed the curve-shaped weld at the face side of the longitudinal attachment and moved into the base metal plate at approximately $45^{\circ}$. Fig. 1 (b) shows 
schematically the path A-A' used for the neutron measurement. Although not shown here, the fatigue crack paths of the two samples were very similar to each other. The "medium crack" $(\mathrm{N}=3 \mathrm{E} 5)$ ended at the weld toe $(11 \mathrm{~mm}$ from the specimen center) while the "long crack" $(\mathrm{N}=4.5 \mathrm{E} 5)$ propagated into the base metal ( $15 \mathrm{~mm}$ from the specimen center).

Fig. 2 (a) shows a micrograph of the weld toe in which the fatigue crack is generated. Fig. 2 (b) illustrates the hardness distribution in the corresponding weld. Vickers hardness measuring (HV1) indicate hardness of approximately $150 \mathrm{HV} 1$ in the base metal and $220 \mathrm{HV} 1$ in the weld metal respectively. Further, this figure illustrates an increased hardness in the HAZ (up to 270 HV1). The high hardness at the weld toe indicates fast cooling after welding. Rapid cooling with cooling times from $800{ }^{\circ} \mathrm{C}$ to $500{ }^{\circ} \mathrm{C}$ in $4 \mathrm{~s}$ to $8 \mathrm{~s}$ is typical for this weld detail and it indicates phase transformation at low temperatures. As mentioned before, phase transformation at low temperatures is associated with a reduction of tensile residual stresses from shrinkage [6].

(a)

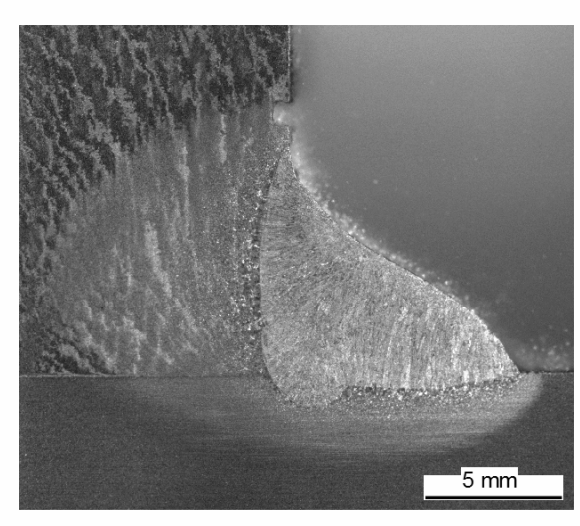

(b)

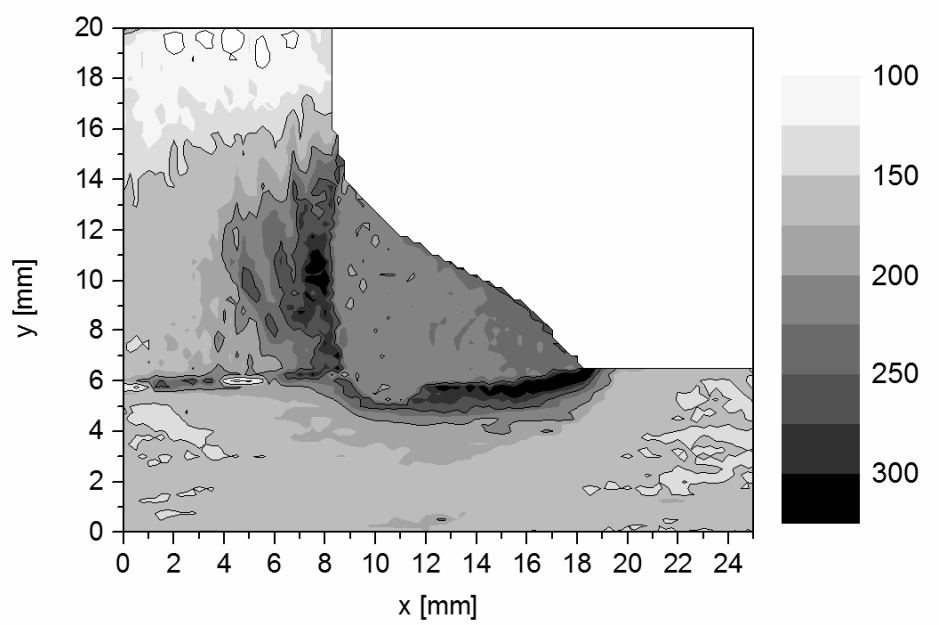

Figure 2 (a) Macrograph of the weld; (b) Hardness distribution of the weld (HV1)

Many engineering disciplines rely on fatigue strength evaluation based on nominal stresses. The fatigue strength evaluation based on nominal stress concepts relies on fatigue testing of small scale specimens. The stress range $\Delta \sigma$ used for testing is plotted over the corresponding number of load cycles $\mathrm{N}$ for a given failure criterion (e.g. specimen fracture or a given crack length). The measure for the fatigue strength is usually the allowable stress range $\Delta \sigma$ at $\mathrm{N}=2 \mathrm{E} 6$ load cycles for a $95 \%$ probability of survival at a given stress ratio of $\mathrm{R}=0.5$ [8]. This fatigue strength in MPa is termed FAT-class. The nominal fatigue resistance at $\mathrm{N}=2 \mathrm{E} 6$ load cycles of this specimen type is FAT56 according to [7] or between FAT63 to FAT71 according to IIW-recommendations [8]. The design FAT-values are valid for a stress ratio of $\mathrm{R}=0.5$ (IIW) and thus not directly comparable to the experimental $\mathrm{S}-\mathrm{N}$ data determined at $\mathrm{R}=-1$ although this specimen type has shown to be independent from mean stress in as welded condition [9].

Experimental S-N data was determined to gain better knowledge about the real fatigue strength of this weld detail in the specific available condition. Fig 3 (a) shows experimental S-N data for this specimen type as well as design S-N curves [8] for the aforementioned FAT-classes. In addition, this Fig. 3 (a) shows two data points of samples containing cracks (without failure) that were used for the neutron diffraction measurement. It can be seen from this plot that the stress range of $\Delta \sigma=120 \mathrm{MPa}$ would lead to fatigue life of about $\mathrm{N}=6 \mathrm{E} 5$ load cycles until specimen failure.

The fatigue cracks in the specimens for the neutron diffraction measurement were detected by means of a simple dye penetration test, Fig. 3 (b). The exact dimensions of the cracks were determined with help of computerized tomography, see Fig. 3 (c). The voxel-size used in the computerized tomography was $0.08 \mathrm{~mm}$. The determined crack dimensions were $23 \mathrm{~mm}$ length and $6 \mathrm{~mm}$ depth (referred as "medium crack") as well as $33 \mathrm{~mm}$ length and $7 \mathrm{~mm}$ depth (referred as "long crack"). 
(a)

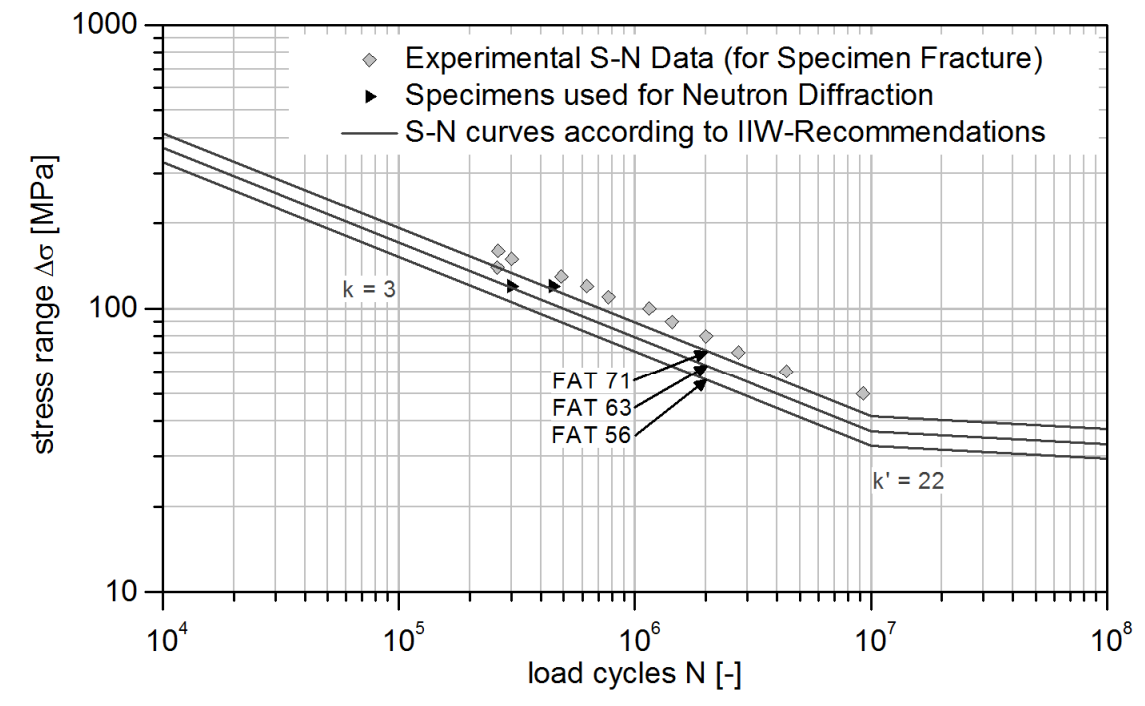

(b)

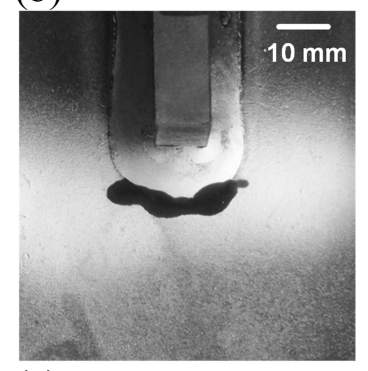

(c)

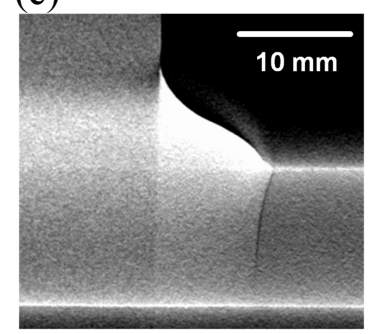

Figure 3 (a) Experimental S-N data $(\mathrm{R}=-1)$ and specimens containing cracks used for neutron diffraction measurement as well as design S-N curves according to [8]; (b) Detection of the fatigue crack at specimen surface by dye penetration test; (b) Computerized tomography through the middle of specimen showing the fatigue crack at the weld toe

Neutron diffraction measurement. The residual stresses have been determined in three welded samples. One of these samples was in the initial "as welded" condition. The others were containing fatigue cracks of different lengths as described above.

Residual stress determination was carried out at the neutron source BER II of the Helmholtz Zentrum Berlin (HZB) using the instrument E3. The paths used for neutron diffraction is shown in Fig. 1 (b). The measurement was started in the middle of the crack at the weld toe and followed the crack along the weld toe into the base metal. The strains at each position were assessed based on the measured diffraction angle $2 \Theta$ in comparison to the stress free reference $2 \Theta_{0}$ from stress relieved free edges. For the measurement $1.5 \times 1.5 \times 2 \mathrm{~mm}^{3}$ was chosen as gauge volume. The first measured volume was placed $0.8 \mathrm{~mm}$ below the upper specimen surface (side with fillet weld). From this point the gauge volume was moved $1 \mathrm{~mm}$ stepwise into sample depth. The distance between two depth-measurement paths along A-A' was $3 \mathrm{~mm}$ along the crack and $1.5 \mathrm{~mm}$ at the crack tip.

Stresses were determined for the loading direction (axial sample direction), perpendicular to the loading direction and in thickness direction. Fig. 4 shows the determined stresses in loading direction for all three specimens. Fig. 4 (a) shows the initial "as welded" residual stresses. The highest residual stresses $(+320 \mathrm{MPa})$ can be seen in a depth of $3 \mathrm{~mm}$. The stresses decrease into depth direction direction as well as to the sides. This area of high tensile residual stresses is where the fatigue crack propagates. Fig. 4 (b) shows the residual stress distribution after $\mathrm{N}=3 \mathrm{E} 5$ load cycles. It can be seen that the fatigue crack propagated approximately $6 \mathrm{~mm}$ into depth. The initial residual stress field was changed significantly. The tensile maximum shifted into a depth of $8 \mathrm{~mm}$ to $9 \mathrm{~mm}$. At the crack front, it formed a tensile residual stress field of about $200 \mathrm{MPa}$. This tensile residual stress field became less distinctive with further crack propagation, Fig. 4 (c).

Not shown here are stresses perpendicular to the loading direction as well as in thickness direction. Perpendicular to the loading direction the tensile residual stress field was also present below the weld in as welded condition. These tensile residual stresses moved with the propagating crack into specimen depth. In thickness direction the residual stresses were low. 
(a) Specimen "as welded"

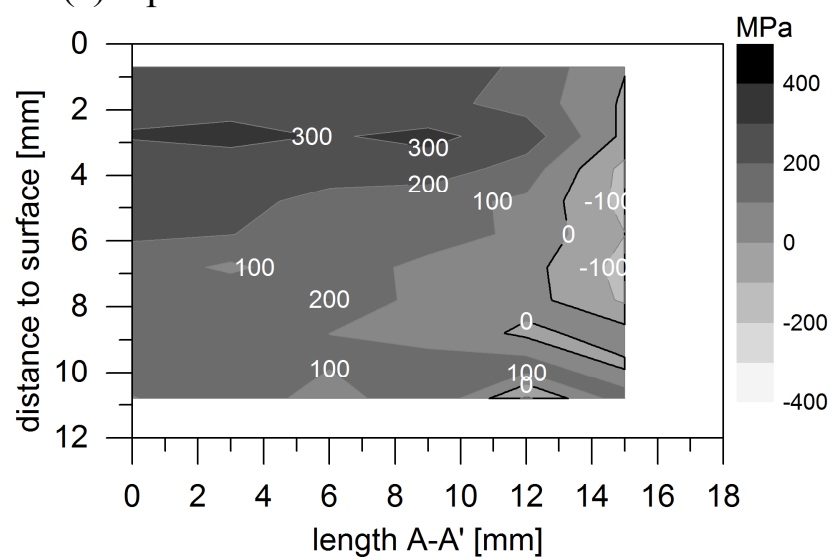

(c) Specimen with "long crack"

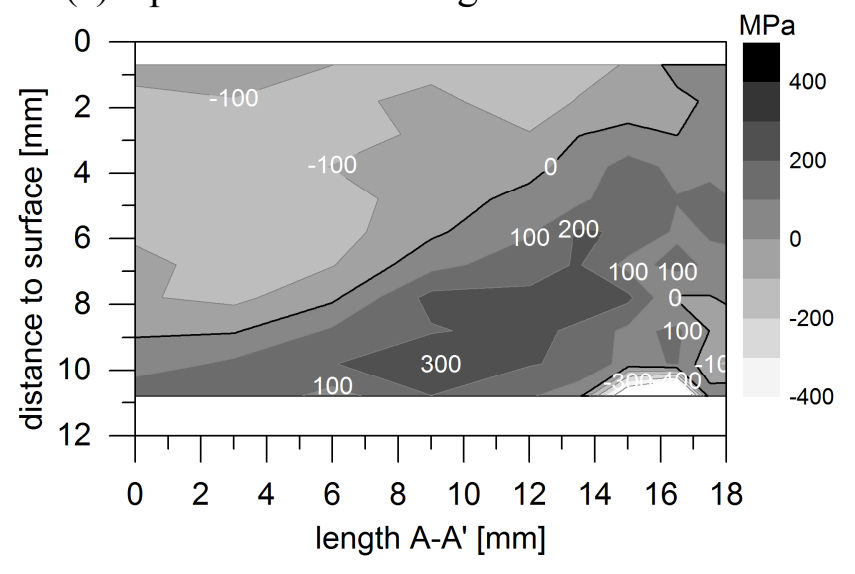

(b) Specimen with "medium crack"

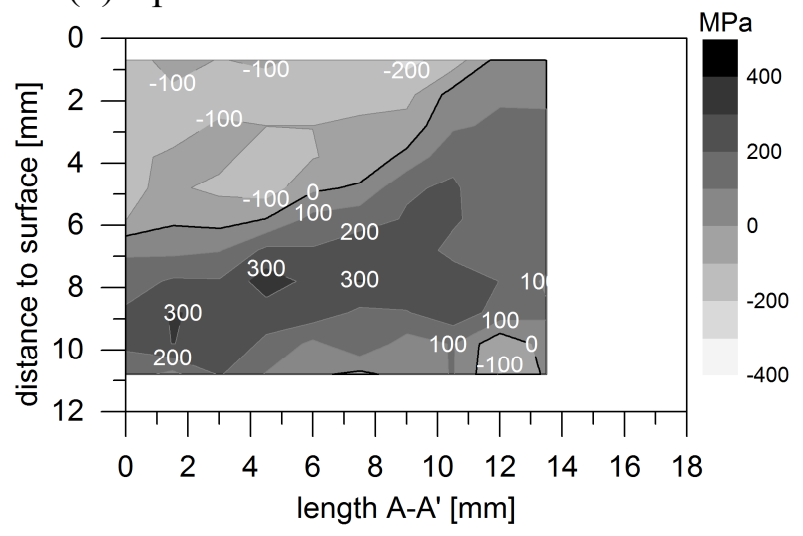

Figure 4 Residual stresses in axial sample direction in non-load carrying fillet welds with different crack lengths. Residual stresses were measured along path A-A' (see Fig. 1) corresponding to the half crack length

\section{Discussion}

In the case of the investigated specimen, the fatigue crack started at the weld toe and propagated through the heat affected zone into the base metal. In these zones different hardness values were observed. The effect of hardness on crack initiation and propagation is not accounted for in this paper but might have significant effect on the crack propagation rate [10]. The fatigue loading initiated macro cracks of several $\mathrm{mm}$ in length. The crack length and the experimental S-N data indicate that the "medium crack" sample was at approximately $50 \%$ of the expectable life time and "long crack" sample was at approximately $75 \%$ respectively.

It was observed that the residual stresses in loading direction showed a tension maximum "as welded" of $320 \mathrm{MPa}$ a few millimeters below the surface. In thickness direction the tensile residual stresses dropped to $100 \mathrm{MPa}$ and they decreased to the sides into compression (-100 MPa at $14 \mathrm{~mm}$ from the center line). The residual stresses redistributed with a growing fatigue crack. In the wake of the crack compressive residual stresses were observed, whereas in front of the crack the tensile residual stresses increased. For example residual stresses increased from $100 \mathrm{MPa}$ ("as welded") up to $300 \mathrm{MPa}$ ("medium crack") in a depth of $8 \mathrm{~mm}$. The compressive residual stresses at the sides of the "as welded" sample changed into tension (100 MPa to $200 \mathrm{MPa})$. These residual tension stresses decreased with ongoing crack propagation ("long crack"). The areas of high tension stresses became smaller (for example into sample depth direction), which may be an effect of the decreasing remaining cross section at the sides of the weld. Initially, the areas beside the weld were in compression balancing the tensile residual stresses below the fillet weld. The redistribution of the residual stresses should be incorporated in case of damage tolerant design where the fatigue crack growth rate is of interest. E.g. [11] show that this effect should be considered in advanced fatigue design concepts.

Future investigations should also incorporate the residual stress redistribution after initial loading or the redistribution in the crack initiation phase. This would allow a better understanding of the 
residual effects on the fatigue in the first half of the expected life time of welded components with high residual stresses.

\section{Summary}

Residual stress fields have been determined in steel plates with longitudinal fillet welded attachments in different conditions; "as welded", "medium crack" and "long crack". The major findings are briefly summarized:

i. The highest residual stresses in loading direction "as welded" were found below the surface and are below the yield strength of the base metal.

ii. The residual stresses redistribute with growing fatigue crack. Compressive residual stresses were observed in the wake of the fatigue crack. In front of the crack tip tensile residual stresses were observed for the "medium crack". In case of the "long crack" almost the entire tensile residual stress field is reduced.

Concluding it can be stated that high tensile residual stresses of approximately $300 \mathrm{MPa}$ (about $70 \%$ of the yield strength) are present in the specimen type investigated in front of the propagating crack. This effect should be considered in crack propagation assessments.

\section{Acknowledgements}

This work is part of the research cluster "IBESS", project G2. The authors gratefully appreciate the funding by the German Research Foundation (DFG).

The neutron diffraction measurement was carried out at the Helmholtz Zentrum für Materialien und Energie, Berlin (HZB). The authors thank for the allocation of beam time (HZB proposal MAT01-3215) and the instrument scientists Dr. R.C. Wimpory and Dr. M. Boin for their professional support.

\section{References}

[1] G. Glinka, Effect of Residual Stresses on Fatigue Crack Growth in Steel Weldments Under Constant and Variable Amplitude Loads, in Fracture Mechanics, ASTM STP 677, C.W. Smith, Ed., American Society for Testing and Materials, 1979, pp. 198-214.

[2] R. Galatolo \& A. Lanciotti, Fatigue Crack Propagation in Residual Stress Fields of Welded Plates, International Journal of Fatigue, Vol. 19, Nr. 1, pp. 43-49, 1997.

[3] C.D.M. Liljedahl, J. Brouard, O. Zanellato, J. Lin, S. Ganguly, P.E. Irving, M.E. Fitzpatrick, X. Zhang and L. Edwards, Weld Residual Stress Effects on Fatigue Crack Growth Behaviour of Aluminium Alloy 2024-T351, International Journal of Fatigue, Vol. 31, pp. 1081-1088, 2009.

[4] C. Liljedahl, O. Zanellato, M. Fitzpatrick, J. Lin and L. Edwards, The Effect of Weld Residual Stresses and their Re-Distribution with Crack Growth during Fatigue under Constant Amplitude Loading, International Journal of Fatigue, Vol. 32, pp. 735-743, 2010.

[5] J. Hensel, T. Nitschke-Pagel, K. Dilger \& S. Schönborn, Effects of Residual Stresses on the Fatigue Performance of Welded Steels with Longitudinal Stiffeners, Materials Science Forum, Vols. 768-769, pp. 636-643, 2013.

[6] H. Wohlfahrt, Die Bedeutung der Austenitumwandlung für die Eigenspannungsentstehung beim Schweißen, Härtereitechnische Mitteilungen, Nr. 41, pp. 248-257, 1986 (in German).

[7] EN1993-1-1, Eurocode 3: Design of Steel Structures, Berlin: Beuth Verlag, 2010.

[8] A. Hobbacher, Recommendations for Fatigue Design of Welded Joints and Components, New York: Welding Research Council, 2009. 
[9] T.R. Gurney, Fatigue of Welded Structures, $2^{\text {nd }}$ Edition, Cambridge University Press, Cambridge, 1979.

[10] E. Macherauch \& W. Reik, Auswirkung von Gefüge, Fertigung und Beanscpruchung auf das Dauerschwingverhalten, in Ermüdungsverhalten metallischer Werkstoffe, Oberursel, Deutsche Gesellschaft für Metallkunde e.V., pp. 111-140, 1985 (in German).

[11] L. Edwards, Influence of Residual Stress Redistribution on Fatigue Crack Growth and Damage Tolerant Design, Materials Science Forum, Vols. 524-525, pp. 363-372, 2006 